\title{
Interface pinning causes the hysteresis of the hydride transformation in binary metal hydrides
}

\author{
Nicholas J. Weadock $\odot,{ }^{1, *}$ Peter W. Voorhees, ${ }^{2,3}$ and Brent Fultz ${ }^{1}$ \\ ${ }^{1}$ Department of Applied Physics and Materials Science, California Institute of Technology, Pasadena, California 91125, USA \\ ${ }^{2}$ Department of Materials Science and Engineering, Northwestern University, Evanston, Illinois 60208, USA \\ ${ }^{3}$ Engineering Sciences and Applied Mathematics, Northwestern University, Evanston, Illinois 60208, USA
}

(Received 10 September 2020; accepted 8 January 2021; published 28 January 2021)

\begin{abstract}
Hydriding and dehydriding transitions in bulk and nanocrystalline binary metal hydrides were studied using the $\mathrm{Pd}-\mathrm{H}$ model system by measuring pressure-composition isotherms with in situ X-ray diffractometry. Nanocrystalline Pd showed a smaller pressure hysteresis, solvus hysteresis, and hysteresis in lattice parameter, compared to bulk Pd. The time-dependence of pressure equilibration was measured after dosing with aliquots of hydrogen, giving equilibration times that were much faster in the single-phase regions than in the two-phase plateaus. In the broad two-phase plateaus, the pressure relaxations were exponential functions of time. An explanation of hysteresis is developed that is based on a dissipative potential barrier that impedes the motion of the interface due to interactions between lattice defects and the two-phase interface. The exponential pressure relaxations and hysteresis are consistent for this mechanism. For a simple model of the pinning potential, the potential barrier maximum is an order of magnitude less than typical grain boundary energies. These pinning effects are substantially different in the nanocrystalline Pd, suggesting differences in the hydriding mechanism.
\end{abstract}

DOI: 10.1103/PhysRevMaterials.5.013604

\section{INTRODUCTION}

Hydrogen-based energy systems exist today and show promise for reducing carbon emissions. Metal hydride systems are now used in battery, fuel cell, thermal storage, and compression technologies [1-5]. One aspect of metal hydrides that lowers their efficiency is hysteresis-the absorption and desorption of hydrogen does not occur with thermodynamic reversibility. This hysteresis has been a research topic for many years. Hysteresis in metal hydrides manifests as a difference in the hydrogen absorption and desorption pressures (pressure hysteresis) and as a difference in the terminal phase boundary compositions during hydriding and dehydriding (solvus hysteresis). The origin of hysteresis remains unclear. We begin by summarizing some important theories of hysteresis that are based on different principles.

Schwarz and Khachaturyan (S-K) presented an analysis of hysteresis that considers how elastic strain energy modifies the thermodynamics of two-phase hydriding and dehydriding phase transitions [6-8]. The S-K theory is based on Eshelby's analysis of elastic inclusions in an infinite matrix. The S-K theory gives the following expression for the pressure hysteresis:

$$
\ln \left(\frac{P_{\mathrm{abs}}}{P_{\mathrm{des}}}\right)=\frac{4 \rho_{\mathrm{H}} Y \eta^{2}\left(c_{\beta}-c_{\alpha}\right)}{k_{\mathrm{B}} T},
$$

where $Y=E /(1-v), E$ is Young's modulus, $v$ is Poisson's ratio, $\eta$ the fractional change in lattice parameter with respect

\footnotetext{
*Present address: SSRL Materials Science Division, SLAC National Accelerator Laboratory, Menlo Park, CA 94025; nweadock@slac.stanford.edu
}

to hydrogen concentration, and $\rho_{\mathrm{H}}$ is a molar volume term which, when fit to molecular dynamics simulations of hydride formation in palladium, was found to be approximately equal to the volume expansion induced by the $\mathrm{H}$ interstitial [8]. The expression also depends on the width of the coherent two-phase region, $c_{\beta}-c_{\alpha}$. The S-K theory assumes coherent interfaces between the matrix and hydride phase; thus elastic energy gives a "macroscopic" nucleation barrier that depends on the hydrogen concentration. In this theory, defects do not relieve the elastic stress.

In an earlier model, Flanagan and Clewley proposed that hysteresis originates with plastic deformation during absorption and desorption [9]. The addition of an enthalpic term corresponding to dislocation formation $\Delta H_{\delta}$ gives a pressure hysteresis of $4 \Delta H_{\delta} / R T$. The addition of $\Delta H_{\delta}$ also manifests in solvus hysteresis. This theory requires a balanced annihilation of dislocations generated during dehydriding, however.

Recently, Griessen et al. developed a mean-field model to predict size-dependent spinodal pressures and associated hysteresis in Pd nanostructures [10]. This model assumes a coherent core-shell hydriding geometry with different hydrogen concentrations in each component. Experimental hysteresis values were reproduced for several Pd nanostructures by assuming a coherent transition with a modified surface-shellcore coupling. An increased solubility of hydrogen in the low-hydrogen-content $\alpha$ phase and reduction in total capacity is attributed to thermodynamically distinct absorption sites on or near nanostructure surfaces [10-12]. However, recent experimental studies demonstrate that spherical cap nuclei are energetically favorable compared to core-shell geometries $[13,14]$.

The palladium-hydrogen system is the prototypical metal hydride and a convenient one for studying hysteresis. At 
moderate temperatures and pressures, fcc palladium absorbs up to $0.7 \mathrm{H}$ per $\mathrm{Pd}$ while undergoing an isostructural expansion from the $\alpha$ phase to the hydrogen-rich $\beta$ phase. This transition results in a volume change of approximately $10 \%$ [15]. Here we characterize the absorption and desorption of hydrogen by $\mathrm{Pd}$ by measuring pressure-composition isotherms with simultaneous in situ x-ray diffraction and by measuring the equilibration times during the individual steps of isotherm measurements. The same hysteresis is found even for incomplete phase transformations - if a partial absorption is reversed, the hydrogen partial pressure shifts to the plateau pressure of the opposite branch before significant changes in hydrogen concentration occur [16]. Hysteresis, relaxation times, and the composition dependencies of lattice parameters are consistent with an explanation for the hysteresis that is based on an interface mobility which is impeded by pinning forces in the microstructure. The pinning potentials are an order of magnitude less than grain boundary energies and vary over a length scale of order $10 \mathrm{~nm}$. The nanocrystalline Pd has crystallite sizes smaller than this, and it has a different hydriding behavior.

\section{INTERFACE MOTION AS THE ORIGIN OF HYSTERESIS}

\section{A. Chemical potential and interface velocity}

Palladium hydrides nucleate coherently. Then, due to their large misfit, they lose coherency as they grow above a critical size $[13,17]$. This loss of coherency creates an array of defects, such as twin boundaries, slip bands, dislocations, and possibly, point defects [17]. The resulting dislocation densities can be quite high, so after the earliest stages of growth, the hydride-matrix interfaces propagate through arrays of defects. Due to elastic and plastic interactions between the defects and interface, a driving force is needed for the interface to move. Such impediments to interface motion have been observed, for example, during martensitic transformations where the interfaces are moving through an array of dislocations $[18,19]$.

We propose that interface pinning causes the hysteresis between absorption and desorption. Assume a planar interface with a local normal velocity of the interface $v$ is related to the driving force for interface migration [20],

$$
v=-M\left\{\llbracket \Omega_{\mathrm{V}} \rrbracket-T_{i j}^{\beta} n_{j} \llbracket E_{i k} \rrbracket n_{k}+\partial \phi_{\mathrm{p}} / \partial x\right\},
$$

where $\llbracket \xi \rrbracket \triangleq \xi^{\beta}-\xi^{\alpha}$ for a quantity $\xi, x$ is a displacement of the interface along the normal direction, $M$ is the mobility of the interface, $\Omega_{\mathrm{V}}$ is the grand potential density, $T_{i j}$ is the stress tensor, $n_{j}$ is the normal to the interface pointing from the $\beta$ phase to the $\alpha$ phase, $E_{i j}$ is the total strain tensor, $\phi_{\mathrm{p}}$ is the pinning potential on the interface caused by defects in the matrix, and summation from 1 to 3 over repeated indices is assumed. The second term on the right-hand side comes from the work required to keep the interface coherent during growth, which we keep at this point to illustrate the potential role of coherency-generated stresses.

If the quantity in the large braces in Eq. (2) is negative, then the $\beta$ phase will grow from $\alpha$. In the absence of stress and pinning, the jump in grand potential is the chemical driving force for interface motion; if the grand potential of $\beta$ is less than that for $\alpha$, the $\beta$ phase will grow. The $\partial \phi_{\mathrm{p}} / \partial x$ is chosen so that when it is positive, it impedes the motion of $\beta$ growing into $\alpha$. We assume that the diffusion of $\mathrm{H}$ is very fast, and its concentration is in local equilibrium at the moving interface. Thus gradients in $\mathrm{H}$ composition within a single phase are small, as observed experimentally, and there is no jump in the diffusion potential of $\mathrm{H}$ at the interface,

$$
\llbracket M_{\mathrm{HV}} \rrbracket=0,
$$

where $M_{\mathrm{HV}}$ is the diffusion potential.

Since $\mathrm{H}$ is an interstitial atom in the Pd lattice, it is necessary to account for the constraint that $N_{I}=N_{\mathrm{H}}+N_{\mathrm{V}}$, where $N_{I}$ is the number of interstitial sites, $N_{\mathrm{H}}$ is the number of $\mathrm{H}$ atoms, and $N_{\mathrm{V}}$ is the number of vacancies on the $\mathrm{H}$ sublattice. The diffusion potential is [20]

$$
M_{\mathrm{HV}}=\rho_{0}\left[\mu_{\mathrm{H}}(c)-\mu_{\mathrm{V}}(c)\right]-\eta T_{k k},
$$

where $\mu_{\mathrm{H}}$ and $\mu_{\mathrm{V}}$ are the stress-free chemical potentials of $\mathrm{H}$ and vacancies, respectively, $\rho_{0}$ is the molar density of interstitial lattice sites, and $\eta$ is the solute expansion coefficient, where $\eta=(1 / a) d a / d c$ ( $a$ is the lattice parameter and $c$ is the mole fraction of $\mathrm{H}$ ). We assume Vegard's law is valid and thus $\eta$ is a constant. The molar grand potential for each phase is [20]

$$
\Omega=F(c)+\rho_{0}^{-1} W_{\mathrm{e}}-M_{\mathrm{HV}} c,
$$

where the molar Helmholtz free energy at zero pressure in the absence of stress is $F=\mu_{\mathrm{H}} c+\mu_{\mathrm{V}}(1-c)$, and $W_{\mathrm{e}}$ is the elastic energy density.

Using Eqs. (4) and (5) in Eqs. (2) and (3), the velocity of the interface is

$$
\begin{aligned}
v= & -M\left\{\rho_{0} \llbracket \mu_{\mathrm{V}}(c) \rrbracket\right. \\
& \left.+\llbracket W_{\mathrm{e}} \rrbracket+\eta \llbracket c T_{k k} \rrbracket-T_{i j}^{\beta} n_{j}^{\beta} \llbracket E_{i k} \rrbracket n_{k}^{\beta}+\partial \phi_{\mathrm{p}} / \partial x\right\}
\end{aligned}
$$

and the diffusion potential is

$$
M_{\mathrm{HV}}=\rho_{0} \llbracket \mu_{\mathrm{H}}(c)-\mu_{\mathrm{V}}(c) \rrbracket-\eta \llbracket T_{k k} \rrbracket=0 .
$$

To define the compositions at the interface, it is necessary to determine the stress in the system. We employ a parallelplate geometry of $\alpha$ and $\beta$ phases in a linearly elastic isotropic solid in which the elastic constants of the two phases are identical and constant. The stress is generated because the compositions of the two phases are different and thus so are their lattice parameters. For a coherent system, the elastic energy and trace of the stress can be determined [21],

$$
\begin{gathered}
\llbracket T_{k k} \rrbracket=-2 Y \eta\left(e_{\mathrm{e}}+\eta \llbracket c-c_{\mathrm{e}} \rrbracket\right), \\
\llbracket W_{\mathrm{e}} \rrbracket+\eta \llbracket c T_{k k} \rrbracket+T_{i j}^{\beta} n_{j}^{\beta} \llbracket E_{i k} \rrbracket n_{k}^{\beta} \\
=Y e_{\mathrm{e}}^{2}-2 \eta e_{\mathrm{e}} Y c_{\mathrm{e}}^{\beta}-2 \eta^{2} Y \llbracket c_{\mathrm{e}}\left(c-c_{\mathrm{e}}\right) \rrbracket,
\end{gathered}
$$

where $c_{\mathrm{e}}^{\alpha}, c_{\mathrm{e}}^{\beta}$ are the equilibrium, stress-free compositions without a pinning potential. The misfit between the two phases is $e_{\mathrm{e}}=\eta \llbracket c \rrbracket$, and the lattice parameter of the $\alpha$ phase at $c_{\mathrm{e}}^{\alpha}$ is used as the reference state for strain. Using Eqs. (8) and (9) in Eqs. (6) and (7) yields the velocity

$$
\begin{aligned}
v= & -M\left\{\rho_{0} \llbracket \mu_{\mathrm{V}}(c) \rrbracket+Y e_{\mathrm{e}}^{2}-2 \eta e_{\mathrm{e}} Y c_{\mathrm{e}}^{\beta}-2 \eta^{2} Y \llbracket c_{\mathrm{e}}\left(c-c_{\mathrm{e}}\right) \rrbracket\right. \\
& \left.+\partial \phi_{\mathrm{p}} / \partial x\right\}
\end{aligned}
$$


and the diffusion potential condition

$$
\rho_{0} \llbracket \mu_{\mathrm{H}}(c)-\mu_{\mathrm{V}}(c) \rrbracket-2 Y \eta^{2} \llbracket c \rrbracket=0 .
$$

These two equations, along with the stress-free chemical potentials (for example, those giving the miscibility gap in the Pd-H system), yield two nonlinear equations for the two interfacial compositions for a given velocity and mobility of the interface. Since the elastic state of one phase is independent of the other, the elastic energies do not depend on the volume fraction of the phases and thus there is no elasticstress-generated metastability and nucleation barrier [21-24].

\section{B. Phase compositions, gas pressures}

When the interface is pinned, $v=0$, and thus the terms in the brackets on the RHS of Eq. (10), along with Eq. (11) give the concentrations at the interface. These concentrations then define the diffusion potential in the solid, which is equal to the chemical potential of $\mathrm{H}$ in the gas. For an approximation that captures the essential physics of the pinning process, the two equations defining the concentrations are solved in the limit that the concentrations of each phase are close to the equilibrium, stress-free compositions without pinning, $c_{\mathrm{e}}^{\alpha}, c_{\mathrm{e}}^{\beta}$. These compositions give the equilibrium incoherent (stress-free, pinning-free) phase diagram. In this limit, the concentration dependence of the terms involving the chemical potentials are given by Taylor expansions about the stress-free equilibrium state of each phase. It is also necessary to linearize the terms involving the stress to first order in $c-c_{\mathrm{e}}$. Using linearized chemical potentials and the linearized forms of the elastic terms yields two linear equations for the two unknown compositions of the phases that give the phase compositions at which the interface is pinned, see Supplemental Materials [25]:

$$
\begin{aligned}
c_{p}^{\alpha}-c_{\mathrm{e}}^{\alpha} & =\frac{\partial \phi_{\mathrm{p}} / \partial x+Y \eta^{2} \llbracket c_{\mathrm{e}} \rrbracket^{2}}{\rho_{0} \llbracket c_{\mathrm{e}} \rrbracket G_{m}^{\prime \prime \alpha}\left(1+B^{\alpha}\right)}, \\
c_{p}^{\beta}-c_{\mathrm{e}}^{\beta} & =\frac{\partial \phi_{\mathrm{p}} / \partial x-Y \eta^{2} \llbracket c_{\mathrm{e}} \rrbracket^{2}}{\rho_{0} \llbracket c_{\mathrm{e}} \rrbracket G_{m}^{\prime \prime \beta}\left(1+B^{\beta}\right)},
\end{aligned}
$$

where $B^{i}=2 \eta^{2} Y / \rho_{0} G_{m}^{\prime \prime i}$ and $G_{m}^{\prime \prime i}=\partial^{2} G_{m}^{i} / \partial c^{2}, i=\alpha, \beta$, evaluated at the equilibrium incoherent compositions of the phases. Since we have assumed that the diffusion potentials are constant, these different compositions give the same diffusion potential in the two-phase mixture.

The solid is in equilibrium with the gas,

$$
\mu_{\mathrm{H}}^{g}=M_{\mathrm{HV}}\left(c, T_{k k}\right),
$$

where $\mu_{\mathrm{H}}^{g}$ is the chemical potential of $\mathrm{H}$ in the gas. For small deviations in the gas pressure about the equilibrium pressure in the absence of stress and pinning, and using Eq. (12) to determine the diffusion potential $M_{\mathrm{HV}}$, the deviation of the equilibrium pressure $P$ at which $v=0$ from the equilibrium value $P_{\mathrm{e}}$ is

$$
\ln \left(\frac{P}{P_{\mathrm{e}}}\right)=\frac{2 \partial \phi_{\mathrm{p}} / \partial x+2 Y \eta^{2} \llbracket c_{\mathrm{e}} \rrbracket^{2}}{\rho_{0} R T \llbracket c_{\mathrm{e}} \rrbracket},
$$

where $R$ is the gas constant and the chemical potential of the gas is $\mu_{\mathrm{H}}^{g}=\mu_{o}+(R T / 2) \ln P, \mu_{o}$ is the standard state chemical potential, and $P$ is the partial pressure of $\mathrm{H}_{2}$ gas. The equilibrium pressure is shifted by the presence of stress but does not change on charging and discharging, and coherencyinduced stress does not cause hysteresis.

\section{Thermodynamics with interface pinning}

The pinning potential causes a hysteresis of absorption and desorption when the pinning force changes sign with the direction of $v$. To illustrate, consider a pinning potential with a periodic dependence,

$$
\phi_{\mathrm{p}}=\frac{\mathcal{A}}{2}[1-\cos (k x)],
$$

where $\mathcal{A}$ sets the strength of pinning, and $k=2 \pi / L$ is the inverse length scale of the interaction. With a greater driving force, the interface is displaced from its equilibrium value at the bottom of the well located at $x=0$. The direction of displacement changes with absorption ( $\beta$ phase grows) or desorption ( $\alpha$ phase grows). The maximum force acting on the interface is given by the interface displacement at which

$$
\frac{\partial^{2} \phi_{\mathrm{p}}}{\partial x^{2}}=0
$$

which is $x_{c}= \pm \frac{L}{2}(n+1 / 2), \quad n=0, \pm 1, \pm 2, \ldots$ Using Eq. (15) and $x_{c}$ gives the maximum pinning force $F_{\mathrm{p}, \mathrm{m}}$ as

$$
F_{\mathrm{p}, \mathrm{m}}=-\left.\frac{\partial \phi_{\mathrm{p}}}{\partial x}\right|_{\max }= \pm \frac{\mathcal{A} \pi}{L}
$$

The plus is the maximum force when charging ( $\beta$ grows) and the minus is the maximum force for discharging ( $\alpha$ grows). The pressure hysteresis is evaluated by subtracting desorption from absorption in Eq. (14):

$$
\ln \left(\frac{P_{\mathrm{abs}}}{P_{\mathrm{des}}}\right)=2 \frac{\left.\left.\partial \phi_{\mathrm{p}} / \partial x\right)_{\mathrm{abs}}-\partial \phi_{\mathrm{p}} / \partial x\right)_{\mathrm{des}}}{\rho_{0} R T \llbracket c_{\mathrm{e}} \rrbracket} .
$$

Thus there is no effect of coherency stress on the hysteresis, since the equilibrium pressure due to coherency stress does not depend on whether hydrogen is being absorbed or desorbed. Using the maximum pinning force derived from Eq. (15),

$$
\ln \left(\frac{P_{\mathrm{abs}}}{P_{\mathrm{des}}}\right)=\frac{4 \mathcal{A} \pi}{L \rho_{0} R T \llbracket c_{\mathrm{e}} \rrbracket} .
$$

The pinning also causes different phase compositions for absorption and desorption. Using Eq. (17) in Eq. (12) yields the pinning compositions of the $\alpha$ phase on absorption and desorption,

$$
\begin{aligned}
c_{\mathrm{abs}}^{\alpha}-c_{\mathrm{e}}^{\alpha} & =\frac{\mathcal{A} \pi / L+Y \eta^{2} \llbracket c_{\mathrm{e}} \rrbracket^{2}}{\llbracket c_{\mathrm{e}} \rrbracket \rho_{0} G_{m}^{\prime \prime^{\alpha}}\left(1+B^{\alpha}\right)}, \\
c_{\mathrm{des}}^{\alpha}-c_{\mathrm{e}}^{\alpha} & =\frac{-\mathcal{A} \pi / L+Y \eta^{2} \llbracket c_{\mathrm{e}} \rrbracket^{2}}{\llbracket c_{\mathrm{e}} \rrbracket \rho_{0} G_{m}^{\prime \prime \alpha}\left(1+B^{\alpha}\right)},
\end{aligned}
$$

expressions that are valid away from the critical temperature, and coherent spinodal.

We expect that the pinning potential $\phi_{\mathrm{p}}(x)$ will be more complicated than Eq. (15), so in this case we can use a general form as a Fourier transform,

$$
\phi_{\mathrm{p}}(x)=\int_{-\infty}^{\infty} \psi_{\mathrm{p}}(k) e^{i k x} d k
$$


The pinning force $F_{\mathrm{p}}(x)$ is thus

$$
F_{\mathrm{p}}(x)=-\frac{\partial \phi_{\mathrm{p}}}{\partial x}=-\int_{-\infty}^{\infty} i k \psi_{\mathrm{p}}(k) e^{i k x} d k
$$

Equation (15) is symmetrical in $\pm x$, so its pinning force $F_{\mathrm{p}}(x)$ is real, and $F_{\mathrm{p}}(x)=-F_{\mathrm{p}}(-x)$, a reasonable but not clearly necessary condition. Equation (22) nevertheless shows that the higher Fourier components of $\psi_{\mathrm{p}}(k)$ are weighted by $k$ and make larger contributions to the pinning force. The spatial range of the pinning force is therefore $L \simeq 2 \pi / k_{\max }$, where $k_{\max }$ is the largest $k$ for which $\psi_{\mathrm{p}}(k)$ is substantial. Equivalently, with a distribution of defects that contribute to $\phi_{\mathrm{p}}(x)$, the effective pinning range $L$ is that of the smallest features that offer substantial pinning.

\section{Kinetics with interface pinning}

We assume that $\mathrm{H}$ diffusion in the $\mathrm{Pd}$ is fast on the timescale of interface motion. When a small amount of $\mathrm{H}$ is added to the surrounding gas, it rapidly enters the Pd, leading to the motion of the interfaces in the two-phase mixture until the diffusion potential in the material is again equal to the chemical potential of the $\mathrm{H}$ gas. The decay of gas pressure can be measured experimentally and used as a probe of the dynamics of interface motion on the two-phase mixture. The velocity of the interface is given by Eq. (6). Assuming small departures in equilibrium from the pinned state, see Supplemental Materials [25],

$$
v=\rho_{0} M\left(1+B^{\alpha}\right) G_{m}^{\prime \prime \alpha} \llbracket c_{\mathrm{p}} \rrbracket\left(c^{\alpha}-c_{\mathrm{p}}\right) .
$$

Defining $f \triangleq V^{\beta} / V_{\mathrm{s}}$ as the volume fraction of $\beta$ phase ( $V_{\mathrm{s}}$ is the total volume of the material system), the rate of change of $f$ is given by

$$
\frac{d V^{\beta}}{d t}=V_{\mathrm{s}} \frac{d f}{d t}=\int_{A} v d s,
$$

where $A$ is the area of the interface. Using Eq. (23) in Eq. (24),

$$
V_{\mathrm{s}} \frac{d f}{d t}=\rho_{0} M\left(1+B^{\alpha}\right) G_{m}^{\prime \prime \alpha} \llbracket c_{\mathrm{p}} \rrbracket \int_{A}\left(c^{\alpha}-c_{\mathrm{p}}^{\alpha}\right) d s .
$$

Since the composition of the $\alpha$ phase is constant along the interface,

$$
\frac{d f}{d t}=\rho_{0} M\left(1+B^{\alpha}\right) G_{m}^{\prime \prime \alpha} \llbracket c_{\mathrm{p}} \rrbracket A\left(c^{\alpha}-c_{\mathrm{p}}^{\alpha}\right) V_{\mathrm{s}} .
$$

Defining the interfacial area per volume $S_{V} \triangleq A / V_{\mathrm{s}}$,

$$
\frac{d f}{d t}=\rho_{0} M\left(1+B^{\alpha}\right) G_{m}^{\prime \prime \alpha} \llbracket c_{\mathrm{p}} \rrbracket S_{V}\left(c^{\alpha}-c_{\mathrm{p}}^{\alpha}\right)
$$

The $S_{V}$ should not vary much after an aliquot of $\mathrm{H}$ is added, but it might change along the isotherm, in the early or late stages of absorption, for example.

After inserting a small aliquot of $\mathrm{H}$, the composition of the material system is $c_{\mathrm{s}}$, with $\mathrm{H}$ distributed between the $\alpha$ and $\beta$ phases:

$$
c_{s}=(1-f) c^{\alpha}+f c^{\beta} .
$$

Since $c_{s}$ is constant after the aliquot of $\mathrm{H}$ is added, for small supersaturations (see Supplemental Materials [25]),

$$
\frac{d c^{\alpha}}{d t}=-\frac{G_{m}^{\prime \prime \beta}\left(1+B^{\beta}\right) \llbracket c_{p} \rrbracket}{G_{m}^{\prime \prime \beta}\left(1+B^{\beta}\right)\left(1-f_{o}\right)+G_{m}^{\prime \prime \alpha}\left(1+B^{\alpha}\right) f_{o}} \frac{d f}{d t},
$$

where $f_{o}$ is the volume fraction before the aliquot $\mathrm{H}$ is added.

Using Eq. (27) in Eq. (29) yields

$$
\frac{d c^{\alpha}}{d t}=-\Lambda M S_{V}(t)\left(c^{\alpha}-c_{\mathrm{p}}^{\alpha}\right),
$$

where $\Lambda$ involves a volume fraction weighted average of thermodynamic terms of each phase,

$$
\Lambda=\frac{\rho_{0} \llbracket c_{p} \rrbracket^{2} G_{m}^{\prime \prime \alpha}\left(1+B^{\alpha}\right) G_{m}^{\prime \prime \beta}\left(1+B^{\beta}\right)}{G_{m}^{\prime \prime \alpha}\left(1+B^{\alpha}\right) f_{o}+G_{m}^{\prime \prime \beta}\left(1+B^{\beta}\right)\left(1-f_{o}\right)} .
$$

Assuming that $S_{V}$ is little changed when a small aliquot of $\mathrm{H}$ is added,

$$
c^{\alpha}(t)=c_{\mathrm{p}}^{\alpha}+\left(c_{\mathrm{pi}}^{\alpha}-c_{\mathrm{p}}^{\alpha}\right) \exp \left\{-\Lambda M S_{V}^{o} t\right\},
$$

where $c_{\mathrm{pi}}^{\alpha}$ is the initial pinned composition, which does not have to be the same after the first absorption because the defects responsible for the pinning may be different, and $S_{V}^{o}$ is the surface area per volume before the aliquot is added.

Since the change in composition in the $\alpha$ phase fixes the diffusion potential, which equals the chemical potential in the gas, Eq. (32) yields

$$
P(t)=P_{\mathrm{p}}+P_{\delta} \exp \left\{-\Lambda M S_{V}^{o} t\right\},
$$

where $P_{\mathrm{p}}$ is the pinning pressure and the transient pressure excursion is $P_{\delta}=\left[\rho_{0}\left(c_{\mathrm{pi}}^{\alpha}-c_{\mathrm{p}}^{\alpha}\right)\left(1+B^{\alpha}\right) G_{m}^{\prime \prime \alpha} P_{\mathrm{p}}\right] /\left[k_{B} T\right]$ and assuming that $\left|P(t)-P_{\mathrm{p}}\right|$ is small. We have allowed for $S_{V}$ to depend on the initial volume fraction $f_{o}$, so there may be different relaxation times across an isotherm.

\section{METHODS}

Palladium powder (200 mesh, 99.95\% metals basis) was purchased from Alfa Aesar (Ward Hill, MA, USA). The powder was annealed at $1273 \mathrm{~K}$ for one hour under $\mathrm{N}_{2}$ flow in a horizontal tube furnace before any hydriding experiments. Palladium nanopowder $(99.95 \%)$ was purchased from US Research Nanomaterials, Inc., (Houston, TX, USA) and degassed at $353 \mathrm{~K}$ for at least 8 hours prior to any hydriding experiments.

Electron micrographs of annealed bulk Pd powder were acquired with a high-resolution Zeiss 1550VP field emission scanning electron microscope (SEM). Nanocrystalline Pd powder was analyzed with bright- and dark-field transmission electron microscopy (TEM) using an FEI Tecnai F-30UT STEM. The powder was dispersed in isopropanol and sonicated one hour to reduce agglomeration before loading on an amorphous carbon grid for TEM.

In situ X-ray diffraction (XRD) experiments were performed using an Inel CPS 120 powder diffractometer utilizing Mo $\mathrm{K} \alpha$ radiation. A $\mathrm{Si}\langle 110\rangle$ single crystal oriented in the $\langle 220\rangle$ direction was used as the incident beam monochromator. Two-theta calibration of the CPS 120 detector was performed with a NIST SRM 660a $\left(\mathrm{LaB}_{6}\right)$. At least $200 \mathrm{mg}$ of sample was first loaded into a temperature-controlled vertical 
sample holder and then placed into a stainless-steel chamber $(980 \mathrm{~mL})$ with a Be window. This reactor is connected to a gas manifold with VCR fittings and MKS Baratron pressure transducers. Prior to hydrogen uptake, the sample was evacuated at $353 \mathrm{~K}$ for $72 \mathrm{~h}$. Each sample was cycled at least once prior to collection of data presented here. Full diffraction measurements from at least two complete isotherms were obtained for each temperature.

Raw diffraction data were initially processed with a deconvolution algorithm to remove instrument effects. Rietveld refinement with the GSAS-II software package was subsequently used to extract lattice parameter data from the deconvoluted in situ XRD results [26]. The Pd-H data were fit with a two-phase model consisting of the solid-solution $\alpha$ phase and the hydride $\beta$ phase, with the sum of the phase fractions constrained to be unity [27].

Pressure-composition isotherms of bulk and nanocrystalline Pd powder were also measured on an independent volumetric Sieverts-type apparatus. At least $1.0 \mathrm{~g}$ of sample were loaded into an AISI 316L stainless-steel reactor $(5 \mathrm{~mL})$ and evacuated (baseline $10^{-5} \mathrm{~Pa}$ ) at $473 \mathrm{~K}$ (bulk Pd) or $353 \mathrm{~K}$ (nanocrystalline Pd) for $8 \mathrm{~h}$. Each sample was cycled at least once prior to collection of data presented here, and at least two complete isotherms were measured for each sample and temperature.

Hydrogen concentrations in the sample were calculated volumetrically with the NIST REFPROP database [28,29]. Absorption and desorption were performed at $333 \mathrm{~K}$ for both the in situ XRD and Sieverts apparatus experiments. An additional pressure-composition isotherm was measured on the Sieverts apparatus for bulk Pd at $435 \mathrm{~K}$. At each absorption (desorption) step, equilibration was reached when pressure in the reactor did not change for a period of $15 \mathrm{~min}$. After equilibration, the next quantity of hydrogen was added (removed for desorption) to the reactor.

\section{RESULTS}

The microstructures of the annealed bulk Pd and asreceived nanocrystalline Pd powders were investigated with scanning electron microscopy (SEM) and transmission electron microscopy (TEM). A high-resolution TEM image of nanocrystalline Pd is provided in Fig. 1, and SEM and additional TEM images are provided in Supplemental Figs. 1 and 2 [25]. Annealed Pd particles are 43.5 $\pm 13 \mu \mathrm{m}$ in size with distinct internal grains of $3.0 \pm 1.3 \mu \mathrm{m}$ in diameter. The nanocrystalline Pd consists of agglomerated crystallites $7.5 \pm 2.3 \mathrm{~nm}$ in diameter, as measured from dark-field images.

Pressure-composition isotherms for bulk and nanocrystalline Pd, measured on a Sieverts apparatus, are shown in Fig. 2(a). Uptake values of approximately 0.6 and $0.7 \mathrm{H} / \mathrm{M}$ are found for the nanocrystalline and bulk Pd, respectively, consistent with other reports $[9,11,12,30-33]$. Defining the dimensionless pressure hysteresis, with $h$ as the left-hand side of Eq. (1),

$$
h=\ln \left(\frac{P_{\mathrm{abs}}}{P_{\mathrm{des}}}\right),
$$

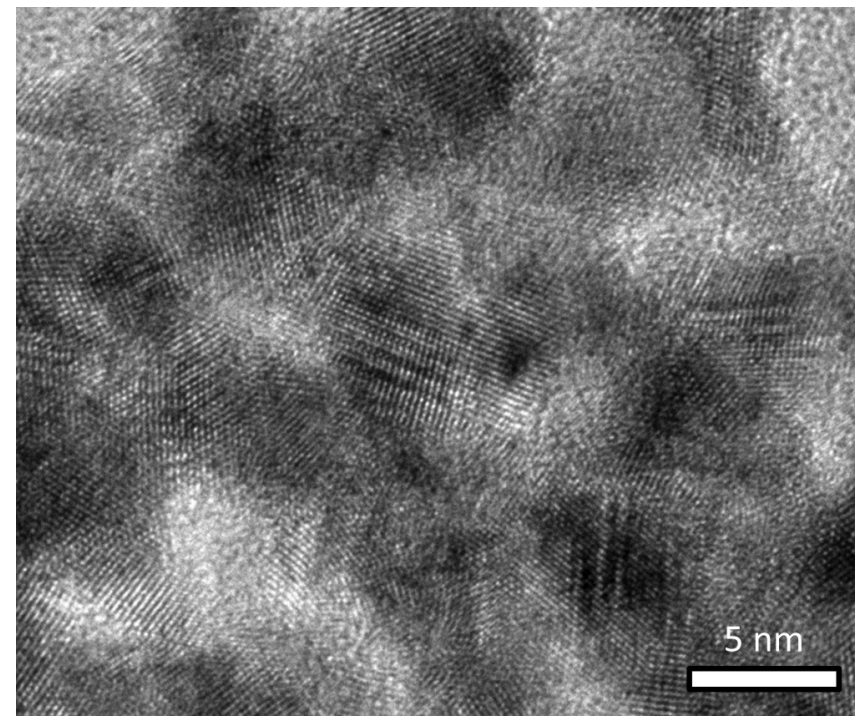

FIG. 1. High-resolution TEM image of the as-received nanocrystalline Pd.

the $333 \mathrm{~K}$ pressure hysteresis measured from the isotherms is 0.37 for nanocrystalline $\mathrm{Pd}$ and 0.88 for bulk $\mathrm{Pd}$. The high-temperature hysteresis of the bulk $\mathrm{Pd}$ at $435 \mathrm{~K}$ is 0.37 . A significant reduction in the absorption plateau pressure for the nanocrystalline Pd (from 10 to $6.9 \mathrm{kPa}$ ) contributes primarily to the reduction in hysteresis at $333 \mathrm{~K}$. Only a small increase in the desorption plateau is observed for the nanocrystalline $\mathrm{Pd}$. Another important difference is that the transition is more gradual for the nanocrystalline Pd; a sharp initial transition is observed for the bulk Pd. We also define solvus hysteresis $\Delta c$ at the $\alpha$ - or $\beta$-phase boundary as

$$
\Delta c^{\alpha}=\left|c_{\mathrm{abs}}^{\alpha}-c_{\mathrm{des}}^{\alpha}\right|,
$$

and similarly for $\Delta c^{\beta}$ for the $\beta$ phase.

Pressure-composition isotherms are also measured independently at $333 \mathrm{~K}$ with a gas manifold and $\mathrm{x}$-ray transparent sample chamber designed for in situ XRD experiments. No in situ data was measured at $435 \mathrm{~K}$ because the hydrogen environment chamber cannot accommodate the required pressures. Diffraction patterns are acquired at several steps along the isotherm, and full sets of diffraction patterns for the hydriding and dehydriding transitions in bulk and nanocrystalline Pd are provided in Supplemental Figs. 4 and 5 [25]. Isotherms measured during the in situ hydriding experiments are consistent with those plotted in Fig. 2(a). They are compared in Supplemental Fig. 3 and discussed in the Supplemental Materials [25]. The hysteresis measured in both apparatus is the same.

Figure 2(b) plots full and minor loop isotherms for Pd$\mathrm{H}$ measured on the in situ system in which absorption was stopped at $75 \%$ completion, then reversed. Rather than traversing back along the absorption branch, the pressure decreases to the desorption branch before significant quantities of hydrogen are removed. Similar results have been reported previously [16].

Lattice parameters obtained from refinement of the in situ diffraction data are plotted in Fig. 3. Variation of lattice pa- 

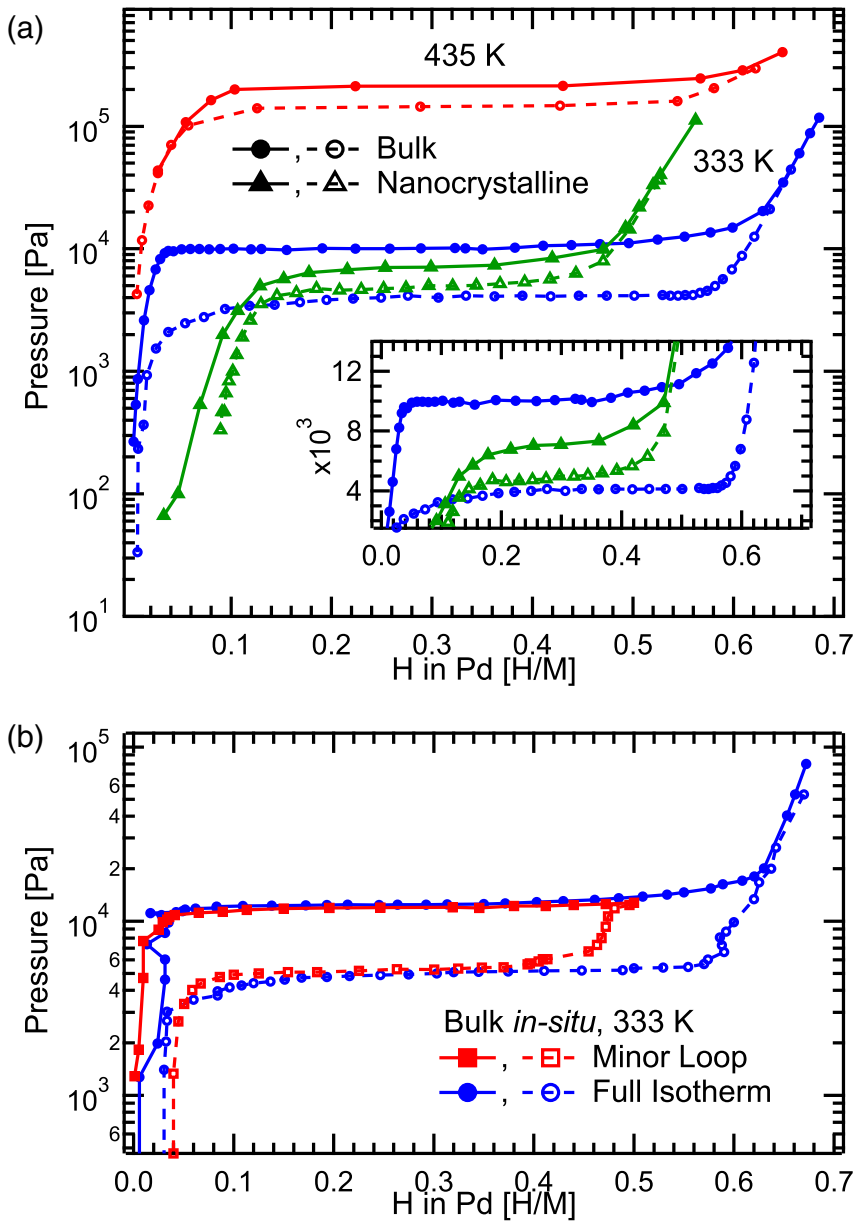

FIG. 2. (a) Pressure-composition isotherms for bulk (blue, red circles) and nanocrystalline (green trianges) Pd measured on a Sieverts apparatus. The inset shows the plateau region on a linear pressure scale. Closed symbols denote absorption, and open symbols correspond to the subsequent desorption. (b) Full and minor loop isotherms for bulk Pd measured in situ. After absorption to approximately $75 \%$ capacity, the isotherm was reversed and the sample was dehydrided.

rameter with hydrogen concentration in single-phase regions was fit to a linear function (Vegard's law); the results are summarized in Table I. Within the two-phase region, lattice parameters are nearly constant but there is variation for the nanocrystalline Pd. This is apparent in the continual increase

TABLE I. Vegard's law relationship and phase boundary compositions in bulk and nanocrystalline $\mathrm{Pd}-\mathrm{H}$ from refined lattice parameter data. The variation of lattice parameter with hydrogen concentration $(1 / a) d a / d c$ is determined in the single-phase regions.

\begin{tabular}{lcc}
\hline Region & Bulk $^{\mathrm{a}}$ & Nanocrystalline \\
\hline$\alpha_{\text {abs }}$ & $0.045 \pm 0.015$ & $0.025 \pm 0.004$ \\
$\alpha_{\text {des }}$ & - & $0.018 \pm 0.011$ \\
$\beta_{\text {abs }}$ & $0.047 \pm 0.013$ & $0.036 \pm 0.003$ \\
$\beta_{\text {des }}$ & $0.039 \pm 0.003$ & $0.039 \pm 0.008$ \\
\hline
\end{tabular}

$\overline{\bar{a} \text { Empty entries correspond to regions with insufficient data points to }}$ fit.
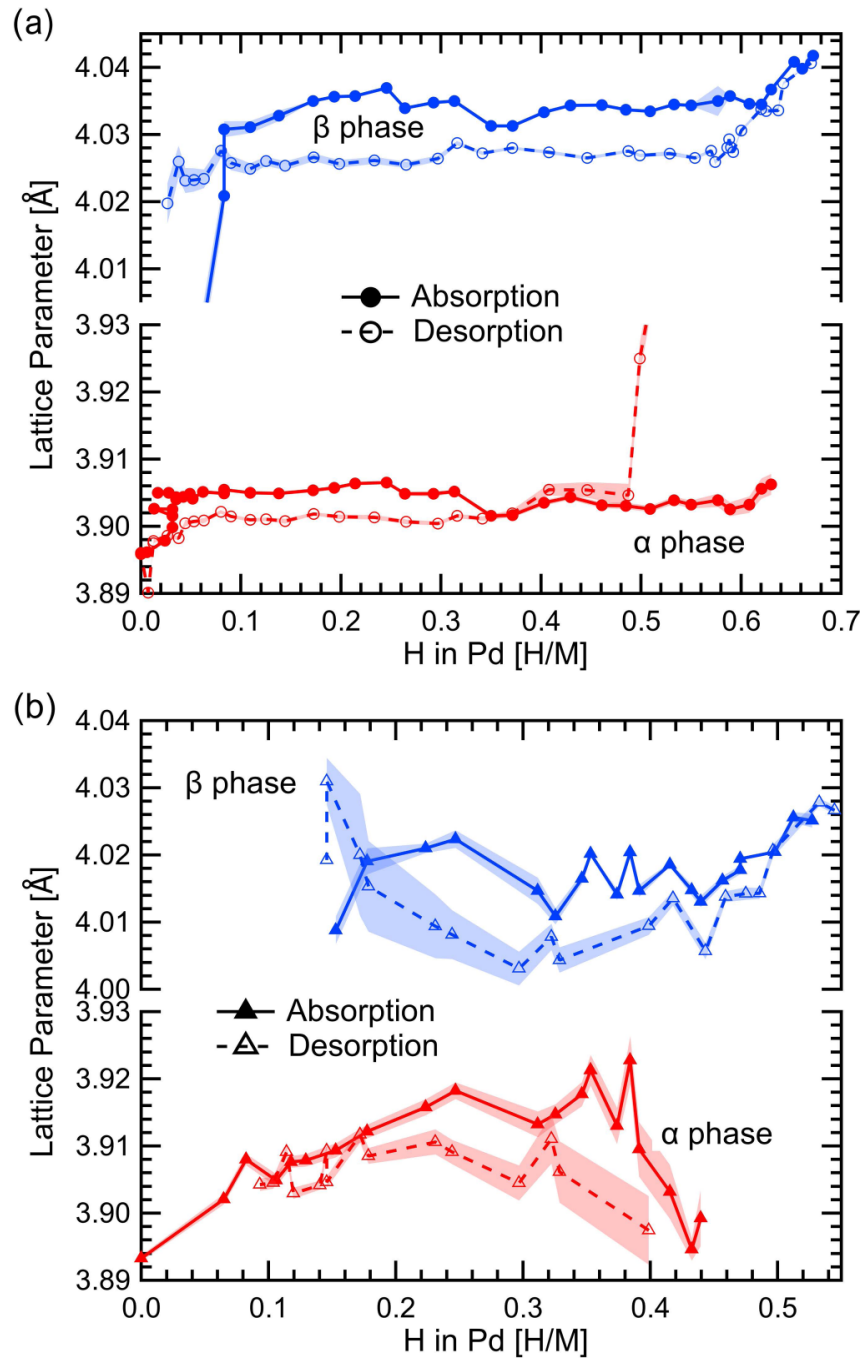

FIG. 3. Refined lattice parameters of the (a) bulk and (b) nanocrystalline $\mathrm{Pd}-\mathrm{H}$ powders during hydrogen absorption and desorption. The red and blue curves correspond to the solid solution $\alpha$ phase and hydride $\beta$ phase, respectively. Error bars are indicated by the shaded region.

in $\alpha$-phase lattice parameter during absorption. Lattice parameters for both phases are larger for absorption than desorption, with a significant difference for bulk Pd [Fig. 3(a)].

In principle, $x$-ray lattice parameters are sensitive to both hydrogen concentration (through Vegard's law) and normal strains from coherency stresses (if present) at $\alpha / \beta$ interfaces. Averaged over the volume of a polycrystalline solid without external forces, the distribution of internal strains generally does not have a substantial effect on the average lattice parameter. In what follows we attribute changes in lattice parameter to changes in hydrogen concentration.

Phase boundary compositions are determined from Fig. 3 by identifying the composition at which the lattice parameters deviate from the values in the two-phase region. These values are reported in Table II. For the nanocrystalline Pd, the $\alpha$-phase boundaries are instead evaluated from the phase fraction results in Supplemental Fig. 6 [25]. In this case the phase boundaries are taken as the concentration in which the phase fraction deviates from the single-phase regime. High- 

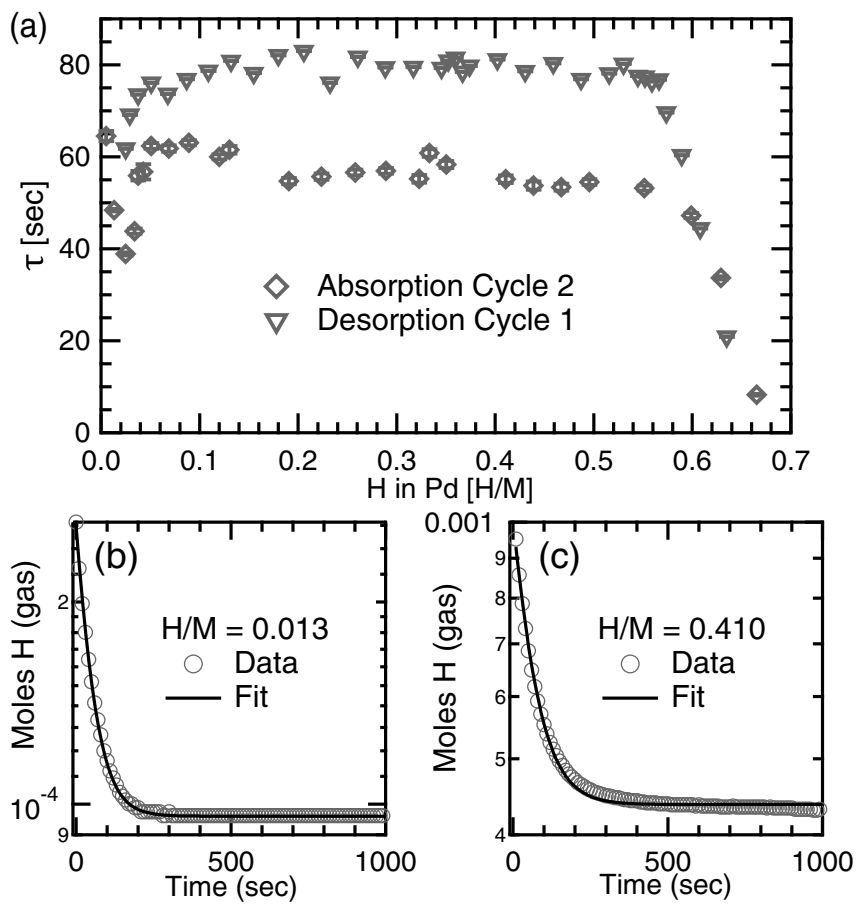

FIG. 4. Kinetics of equilibration during absorption and desorption. (a) Equilibration time constants $\tau$ as a function of hydrogen concentration during cycling. Raw data from absorption cycle 2 and corresponding fits used to extract $\tau$ are plotted in (b) for the single-phase region and in (c) for the two-phase region. Reported error bars may be smaller than the associated data marker.

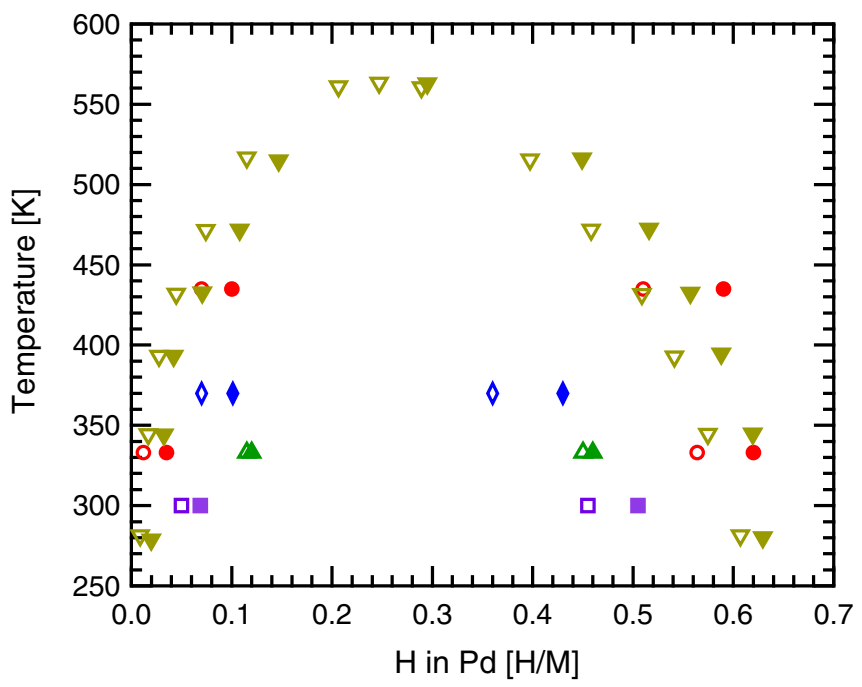

FIG. 5. Absorption and desorption phase boundaries determined from terminal compositions of bulk and nanocrystalline Pd-H. Bulk and nanocrystalline $\mathrm{Pd}-\mathrm{H}$ data from this study are plotted as red circles and green triangles, respectively. Additional bulk data from Wicke et al. is plotted as upside down triangles [32]. 6- and 4-nm nanoparticle Pd data from Vogel et al. is plotted as squares and diamonds, respectively [27]. Filled markers denote absorption, open markers denote desorption.
TABLE II. Terminal compositions and solvus hysteresis of the hydriding transition for bulk and nanocrystalline Pd obtained from in situ XRD results at $333 \mathrm{~K}$. High-temperature (435 K) compositions for the bulk $\mathrm{Pd}$ are evaluated from the pressure-composition isotherm. The solvus hysteresis $\Delta c^{\alpha / \beta}$ are evaluated using Eq. (35).

\begin{tabular}{|c|c|c|c|}
\hline \multirow[t]{2}{*}{ Sample } & \multicolumn{2}{|l|}{ Bulk } & \multirow{2}{*}{$\begin{array}{c}\text { Nanocrystalline } \\
333 \mathrm{~K}\end{array}$} \\
\hline & $333 \mathrm{~K}$ & $435 \mathrm{~K}$ & \\
\hline$c_{\alpha}^{\mathrm{abs}}$ & $0.035 \pm 0.001$ & 0.13 & $0.12 \pm 0.01$ \\
\hline$c_{\beta}^{a}$ & $0.62 \pm 0.01$ & 0.59 & $0.46 \pm 0.015$ \\
\hline$c_{\alpha}^{\mathrm{des}}$ & $0.012 \pm 0.0005$ & 0.07 & $0.115 \pm 0.005$ \\
\hline$c_{\beta}^{\mathrm{des}}$ & $0.564 \pm 0.01$ & 0.51 & $0.45 \pm 0.01$ \\
\hline$\Delta c^{\alpha}$ & $0.023 \pm 0.0011$ & 0.06 & $0.005 \pm 0.011$ \\
\hline$\Delta c^{\beta}$ & $0.056 \pm 0.014$ & 0.08 & $0.01 \pm 0.018$ \\
\hline
\end{tabular}

temperature phase boundary compositions were determined by the graphical method proposed by Frieske and Wicke, in which the plateaus are extended to the opposite isotherm branch and the intersection point is taken as the phase boundary [32]. The solvus hysteresis is evaluated with Eq. (35) and also reported in Table I.

The variation of hydrogen pressure was monitored as a function of time at each absorption or desorption step during pressure-composition isotherm measurements and fit to the exponential function in Eqs. (32) and (33). A characteristic equilibration time $\tau$, corresponding to $\left[\Lambda M S_{V}^{o}\right]^{-1}$, is plotted in Fig. 4(a) as a function of hydrogen content for the first desorption and second absorption cycles. Representative fits to hydrogen absorption in the single- and two-phase regions are plotted in Figs. 4(b) and 4(c), respectively. These fits were also performed for data in the single-phase regions.

Equilibration times increase as the two-phase region is approached from either side. After the transformation enters the two-phase region, however, there is little variation in $\tau$. Hydrogen diffusivity in palladium at $333 \mathrm{~K}$ is $10^{-6} \mathrm{~cm}^{2} \mathrm{~s}^{-1}$, corresponding to diffusion times of $5 \mathrm{~s}$ into the center of bulk $\mathrm{Pd}$ particles [34], and the characteristic time for heating the reactor vessel was measured to be $5.4 \mathrm{~s}$. Therefore $\tau$ is not dominated by the diffusion of hydrogen or heat within the two-phase region. It is possible that the diffusion of hydrogen and heat account for much of the relaxation time in the singlephase regions.

\section{DISCUSSION}

\section{A. Hysteresis and hysteresis energies}

The magnitude of the pinning potential $\mathcal{A}$ is evaluated from Eq. (19) using the experimentally determined pressure hysteresis, $\rho_{0}$, and approximate value of $\llbracket c_{e} \rrbracket$. We assume a characteristic $L=10-35 \mathrm{~nm}$, obtained as the inverse of the reported dislocation densities in cycled Pd-H [35-37]. The pinning potentials for bulk $\mathrm{Pd}-\mathrm{H}$ are $0.1-0.34$ and 0.04 $0.15 \mathrm{~J} \mathrm{~m}^{-2}$ at 333 and $435 \mathrm{~K}$, respectively. These energies are about an order of magnitude less than grain boundary energies calculated for fcc metals $[38,39]$. The solvus hysteresis is different at the $\alpha$ and $\beta$ phase boundaries, as seen in Table II. This behavior is consistent with the predictions of Eqs. (12) and (20), as $G_{m}^{\prime \prime \alpha}$ and $G_{m}^{\prime \prime \beta}$ are not equivalent. 
We next compare our results to the S-K theory, which predicts that hysteresis is a result of an additional energy input needed to overcome a macroscopic coherent nucleation barrier associated with the total elastic strain energy of a mixture of two phases. Thus for the strain state assumed in their work, for a second phase to form an energy barrier must be overcome, and once it does the entire system transforms to the other phase, which occurs with a sufficient change in pressure of the gas. This prediction is not consistent with behavior observed in the minor loop of Fig. 2(b). Both phases are present when the direction of the transformation is reversed, meaning that no nucleation barrier should exist.

The S-K theory also does not quantitatively describe the hysteresis of isotherms of $\mathrm{Pd}-\mathrm{H}$. The $\mathrm{S}-\mathrm{K}$ pressure hysteresis (evaluated in Fig. 14 of [8]) for bulk Pd-H is 4.1 and 2.0 at 333 and $435 \mathrm{~K}$, respectively, and 2.2 for nanocrystalline $\mathrm{Pd}-\mathrm{H}$ at $333 \mathrm{~K}$, using $\left(c_{\beta}-c_{\alpha}\right)$ as determined from our phase fraction data (Table II) $[34,40]$. A shear modulus of $35 \mathrm{GPa}$ is used for the nanocrystalline Pd [41]. The S-K theory significantly overestimates the measured hysteresis of $\mathrm{Pd}-\mathrm{H}$.

Other theories propose that hysteresis is due to continued formation of dislocations as hydriding transitions occur [9]. The energy of these dislocations is analogous to the stored energy of cold work. We define a hysteresis energy, calculated as the difference in diffusion potential between the absorption and desorption plateaus,

$$
\Delta M_{\mathrm{HV}}=\frac{1}{2} R T \rho_{P d} \ln \left(\frac{P_{\mathrm{abs}}}{P_{\mathrm{des}}}\right),
$$

where $\rho_{P d}$ is the molar volume of Pd. From our experimental isotherms, hysteresis energies for bulk $\mathrm{Pd}-\mathrm{H}$ are $1.4 \times 10^{8}$ and $7.6 \times 10^{7} \mathrm{~J} \mathrm{~m}^{-3}$ at 333 and $435 \mathrm{~K}$, respectively. In coldworked fcc metals the stored energy is on the order of $1.0 \times$ $10^{6} \mathrm{~J} \mathrm{~m}^{-3}$, far less than measured hysteresis energies [42].

Equilibration times [Fig. 4(a)] are longer in the two-phase region, indicating the presence of a persistent barrier to phase transformation that does not exist in single-phase regions. When the pinning potential is overcome by a change in hydrogen partial pressure, the system returns to the $v=0$ equilibrium state as a decaying exponential in pressure according to Sec. II D. An exponential decay is seen in the data of Fig. 4(c), but deviations are apparent at long times. These deviations could arise from a distribution of pinning potentials within the material. Interestingly, the observed $\tau$ are different for absorption and desorption, with the difference larger than variations in decay times across the individual transformation. We attribute deviations in $\tau$ to changes in $S_{V}(f)$ during the transformation, although the pinning potential may differ for absorption and desorption. More work is needed to explore these possibilities.

We estimate the unpinned interface velocity using the measured $\tau$. Supplemental Fig. 7 plots the maximum interfacial velocity as a function of $S_{V}(f)$ given $\tau$ [25]. By averaging $\Lambda$ in Eq. (31) and placing bounds on $S_{V}(f)$ as discussed in the Supplemental Materials [25], we expect a maximum velocity on the order of $0.1 \mu \mathrm{m} / \mathrm{s}$, which decays to approximately $1 \mathrm{~nm} / \mathrm{s}$ before the interface is again pinned.

Nanocrystalline Pd-H has a smaller hysteresis, smaller total hydrogen capacity, and narrower two-phase region than bulk Pd-H; see Fig. 2 and Table II. These characteristics are also reflected in the lattice parameters of Fig. 3; during absorption, the lattice parameter of the $\alpha$ phase is greater than in the bulk. During desorption the lattice parameters of the $\beta$-phase are less than the bulk.

The variation of lattice parameter with hydrogen content $(1 / a) d a / d c$ is smaller in nanocrystalline than bulk Pd, especially for the $\alpha$ phase. This means that the volume distortion is less per hydrogen, allowing for increased solubility of hydrogen in the $\alpha$ phase. It has been hypothesized that increased solubility of hydrogen in the $\alpha$ phase and reduction in total capacity is due to a significant fraction of energetically favorable interstitial sites near the particle surface [10-12]. A high density of grain boundaries in nanocrystalline Pd is visible in Fig. 1 that can also adsorb $\mathrm{H}$.

Estimating the pinning potential in nanocrystalline Pd$\mathrm{H}$ using $L$ set by the dislocation density is improper, as (de)hydriding of Pd nanostructures has been demonstrated to occur without plastic deformation below a critical size of $300 \mathrm{~nm}[13,14,43,44]$. Hysteresis increases with particle size up to this critical size, with a maximum value of 1.41 evaluated from data reported by Griessen et al. [10] and Ulvestad et al. [13]. It is likely that the hydriding transition occurs entirely coherently for nanoparticles below $300 \mathrm{~nm}$, yet hysteresis predicted by the S-K theory is still an overestimate.

As stated in Sec. II C, the general pinning potential is set by the largest Fourier coefficient allowed by the geometry of the system. Setting bounds on $L$ and therefore a minimum $k_{\max }$ restricts the possible values of $\psi_{\mathrm{p}}(k)$. Thus, while Eq. (19) does not have an explicit particle size dependence, the inherent size dependence of Eq. (22) is captured in Eq. (18). As a simple example, we use the nanocrystalline $\mathrm{Pd}-\mathrm{H}$ hysteresis at $333 \mathrm{~K}$ to evaluate $\mathcal{A}=0.01 \mathrm{~J} \mathrm{~m}^{-2}$ using Eq. (19) with $L=3.5 \mathrm{~nm}$, half the average particle size. We note that the nanocrystalline Pd used in this work has crystallites smaller than the dislocation separation for bulk Pd-H, indicating dislocations likely do not serve as pinning sites in this system. The magnitude of pinning in nanoscale $\mathrm{Pd}-\mathrm{H}$ is less than in bulk $\mathrm{Pd}-\mathrm{H}$, as evidenced by the reduced hysteresis, but refining the mechanism will require further work to determine the type and energetics of defects present in nanoscale Pd-H.

Additional studies of the hydriding transition in $\mathrm{Pd}-\mathrm{H}$ nanostructures report behavior consistent with our predictions. Sytwu et al. report a constant interface velocity for dislocation-free hydriding transitions in nanostructured $\mathrm{Pd}-\mathrm{H}$, which was not diffusion limited [44]. Furthermore, Ulvestad and Yau observed that the presence of dislocations in Pd nanoparticles impedes the hydriding transition [45]. This behavior is consistent with our model of an interface-based hysteresis and indicates that pinning can originate from more than just dislocations.

\section{B. Chemical variations within the $\alpha$ and $\beta$ phases}

Pressure and solvus hysteresis are both apparent in the hydriding of Pd-H, as observed in Fig. 2 and Table I. Shifts in the phase boundary composition resulting from the solvus hysteresis manifest in the refined lattice parameters of Fig. 3. A reduced hydrogen concentration of each phase during desorption (as compared to absorption) results in smaller lattice parameters of each phase. During hydrogen absorption by 
bulk Pd, $\bar{a}_{\beta}=4.034 \AA$ whereas $\bar{a}_{\beta}=4.025 \AA$ on desorption. A similar trend is observed for the $\alpha$-phase lattice parameters.

The lattice parameter, and therefore hydrogen content, in both phases remains constant during the phase transformation. In the case of hysteresis arising from a macroscopic nucleation barrier, we would expect that supersaturation of the parent phase would result in changes in the lattice parameter as supersaturated hydrogen diffuses to the growing phase. Instead, the constant hydrogen concentration suggests a persistent barrier to growth of a new phase, consistent with interface pinning.

\section{Hysteresis effects on the phase diagram}

Solvus hysteresis alters the measured phase boundaries on the $P-c$ phase diagram. The $\mathrm{Pd}-\mathrm{H}$ phase diagram in Fig. 5 includes data from Table II and from the literature [32]. The phase boundaries for absorption are shifted to the right compared to desorption. Wicke et al. proposed that desorption is closer to true "strain-free" equilibrium, and thus desorption data should be used for determining the true phase boundaries [32]. We accept the existence of strains and stresses of hydriding, but we show in Sec. II B that hydriding stresses do not cause hysteresis, although they shift the equilibrium pressures. With hysteresis, neither absorption nor desorption are representative of either coherent or incoherent phase equilibrium.

With an impediment to interface movement, phase boundaries for neither absorption nor desorption are set by a true minimum of free energy. Nevertheless, phase boundaries measured with interface impediments are the physically relevant phase boundaries. Accessing true equilibrium phase boundaries would require no microstructural barrier to interface movement, as in a material without dislocations or other heterogeneities. This is unlikely, given the large lattice parameter misfit between the phases. Measuring the thermodynamic phase boundary requires that both an incoherent interface exists between the phases, and no dislocations or other defects are present to pin the interface. Such an ideal material is not expected in practice.

\section{CONCLUSIONS}

The equilibrium pressures of hydrogen absorption and desorption in bulk and nanocrystalline $\mathrm{Pd}-\mathrm{H}$ were measured simultaneously with in situ X-ray diffraction patterns. For bulk $\mathrm{Pd}-\mathrm{H}$, the equilibrium pressure and lattice parameter were nearly constant across the two-phase plateau, but the hysteresis in both quantities was large when switching from absorption to desorption. The time response of the pressure was measured for each aliquot of hydrogen added to the material and showed an exponential relaxation that was about $40 \%$ faster for absorption than desorption, but the relaxation time was nearly constant across the two-phase plateau. The time constant was much shorter in the single-phase regions of the $\mathrm{Pd}-\mathrm{H}$ phase diagram.
An explanation of hysteresis is developed, based on a dissipative potential barrier that impedes the motion of the two-phase interface, analogous to a Peierls barrier for defect motion. This potential barrier raises the pressure for absorption and decreases it for desorption. We estimate pinning potentials of order $0.1-0.2 \mathrm{~J} \mathrm{~m}^{-2}$, an order of magnitude smaller than grain boundary energies. An exponential time decay of the applied pressure is consistent with an interface mobility that is driven by a chemical potential with a pinning potential. An interface velocity of order $0.1 \mu \mathrm{m} / \mathrm{s}$ is estimated for the isotherms measured at $333 \mathrm{~K}$, but the precise velocity depends on the density and shape of particles of the new phase. When the interface motion is reversed part way through the two-phase region, the pressure quickly returns to the precise pressure of the other branch of the isotherm. This is consistent with the reversal of the pinning force for interface motion but not with a macroscopic nucleation barrier from elastic energy.

Nanocrystalline Pd shows different hydriding behavior than the bulk, with a smaller hysteresis and smaller hydrogen capacity. The lattice parameters of the two phases of nanocrystalline $\mathrm{Pd}-\mathrm{H}$ differ from the bulk material, indicating that hydrogen absorption and desorption in nanocrystalline $\mathrm{Pd}$ follows a different mechanism. Other metal hydride systems involving anisotropic expansions or structural changes also exhibit hysteresis; further work is necessary to analyze these systems in the framework developed here [46,47].

The phase compositions show a distinct hysteresis, with phase compositions that differ for absorption and desorption. While this is a practical feature of hydriding in a material with microstructural defects, these compositions are not equilibrium ones but are associated with the diffusion potential necessary to overcome the pinning force on the interface. Nevertheless, they are pertinent to the different processes of absorption and desorption.

The analysis of a dissipative potential barrier to interface motion, used here to explain hysteresis in $\mathrm{Pd}-\mathrm{H}$, is not limited to metal hydride phase transitions. This type of barrier should be considered in all solute-driven phase transitions, including those which have become ubiquitous in the energy storage field.

\section{ACKNOWLEDGMENTS}

The authors acknowledge A. Moorthy for assistance with experimental data collection and helpful discussion. We gratefully acknowledge the critical support and infrastructure provided for this work by the Kavli Nanoscience Institute at Caltech. This work was supported by the National Science Foundation under Grant No. 1904714. P.W.V. is grateful for financial support received as a Moore Distinguished Scholar at Caltech. We gratefully acknowledge the critical support and infrastructure provided for this work by The Kavli Nanoscience Institute at Caltech.
[1] H. Yang, N. J. Weadock, H. Tan, and B. Fultz, J. Mater. Chem. A 5, 21785 (2017).
[2] E. C. E. Rönnebro and E. H. Majzoub, MRS Bull. 38, 452 (2013). 
[3] V. A. Yartys, M. Lototskyy, V. Linkov, D. Grant, A. Stuart, J. Eriksen, R. Denys, and R. C. Bowman, Appl. Phys. A 122, 415 (2016).

[4] M. V. Lototskyy, V. A. Yartys, B. G. Pollet, and R. C. Bowman Jr., Int. J. Hydrogen Energy 39, 5818 (2014).

[5] R. C. Bowman and B. Fultz, MRS Bull. 27, 688 (2002).

[6] R. B. Schwarz and A. G. Khachaturyan, Phys. Rev. Lett. 74, 2523 (1995).

[7] R. B. Schwarz and A. G. Khachaturyan, Acta Mater. 54, 313 (2006).

[8] R. B. Schwarz, A. K. Khachaturyan, A. Caro, M. I. Baskes, and E. Martinez, J. Mater. Sci. 55, 4864 (2020).

[9] T. B. Flanagan and J. D. Clewley, J. Less-Common Met. 83, 127 (1982).

[10] R. Griessen, N. Strohfeldt, and H. Giessen, Nat. Mater. 15, 311 (2016).

[11] A. Pundt, C. Sachs, M. Winter, M. T. Reetz, D. Fritsch, and R. Kirchheim, J. Alloys Compd. 293, 480 (1999).

[12] C. Sachs, A. Pundt, R. Kirchheim, M. Winter, M. T. Reetz, and D. Fritsch, Phys. Rev. B 64, 075408 (2001).

[13] A. Ulvestad, M. J. Welland, W. Cha, Y. Liu, J. W. Kim, R. Harder, E. Maxey, J. N. Clark, M. J. Highland, H. You, P. Zapol, S. O. Hruszkewycz, and G. B. Stephenson, Nat. Mater. 16, 565 (2017).

[14] T. C. Narayan, F. Hayee, A. Baldi, A. L. Koh, R. Sinclair, and J. A. Dionne, Nat. Commun. 8, 14020 (2017).

[15] H. W. King and F. D. Manchester, J. Phys. F 8, 15 (1978).

[16] D. Wang, T. B. Flanagan, and T. Kuji, Phys. Chem. Chem. Phys. 4, 4244 (2002).

[17] E. Ho, H. A. Goldberg, G. C. Weatherly, and F. D. Manchester, Acta Metall. 27, 841 (1979).

[18] G. Ghosh and G. B. Olson, Acta Metall. Mater. 42, 3361 (1994).

[19] V. I. Levitas and D.-W. Lee, Phys. Rev. Lett. 99, 245701 (2007).

[20] P. W. Voorhees and W. C. Johnson, Solid State Phys. Adv. Res. Appl. 59, 1 (2004).

[21] W. C. Johnson and C. Chiang, J. Appl. Phys. 64, 1155 (1988).

[22] J. W. Cahn, Acta Metall. 10, 907 (1962).

[23] C. Chiang and W. C. Johnson, J. Mater. Res. 4, 678 (1989).

[24] W. C. Johnson and P. Voorhees, Metall. Trans. A 18, 1213 (1987).

[25] See Supplemental Material at http://link.aps.org/supplemental/ 10.1103/PhysRevMaterials.5.013604 for additional electron microscopy images and X-ray diffraction data, an evaluation of isotherms measured on the in situ environment chamber, and additional derivations for the interface velocity and pressure decay.
[26] B. H. Toby and R. B. Von Dreele, J. Appl. Crystallogr. 46, 544 (2013).

[27] W. Vogel, W. He, Q.-H. Huang, Z. Zou, X.-G. Zhang, and H. Yang, Int. J. Hydrogen Energy 35, 8609 (2010).

[28] E. W. Lemmon, I. Bell, M. L. Huber, and M. O. McLinden, NIST Standard Reference Database 23: Reference Fluid Thermodynamic and Transport Properties-REFPROP, Version 10.0, National Institute of Standards and Technology, Gaithersburg, MD (2018).

[29] J. W. Leachman, R. T. Jacobsen, S. G. Penoncello, and E. W. Lemmon, J. Phys. Chem. Ref. Data 38, 721 (2009).

[30] M. Yamauchi, R. Ikeda, H. Kitagawa, and M. Takata, J. Phys. Chem. C 112, 3294 (2008).

[31] F. Manchester, Phase Diagrams of Binary Hydrogen Alloys, Monograph series on alloy phase diagrams (ASM International, 2000).

[32] E. Wicke and J. Blaurock, J. Less-Common Met., Proceedings of the International Symposium on the Properties and Applications of Metal Hydrides V, 130, 351 (1987).

[33] H. Frieske and E. Wicke, Ber. Bunsen Ges. Phys. Chem. 77, 48 (1973).

[34] Hydrogen in Metals I: Basic Properties, edited by G. Alefeld and J. Völkl, Topics in Applied Physics (Springer-Verlag, Berlin, 1978).

[35] T. B. Flanagan, B. S. Bowerman, and G. E. Biehl, Scr. Metall. 14, 443 (1980).

[36] H. C. Jamieson, G. C. Weatherly, and F. D. Manchester, J. LessCommon Met. 50, 85 (1976).

[37] M. L. H. Wise, J. P. G. Farr, and I. R. Harris, J. Less-Common Met. 41, 115 (1975).

[38] D. L. Olmsted, S. M. Foiles, and E. A. Holm, Acta Mater. 57, 3694 (2009).

[39] V. V. Bulatov, B. W. Reed, and M. Kumar, Acta Mater. 65, 161 (2014).

[40] Smithells Metals Reference Book (Seventh Edition), edited by E. A. Brandes and G. B. Brook (Butterworth-Heinemann, Oxford, 1992), pp. 15-1.

[41] M. Weller, J. Diehl, and H. E. Schaefer, Philos. Mag. A 63, 527 (1991).

[42] M. B. Bever, D. L. Holt, and A. L. Titchener, Prog. Mater. Sci. 17, 5 (1973).

[43] F. Hayee, T. C. Narayan, N. Nadkarni, A. Baldi, A. L. Koh, M. Z. Bazant, R. Sinclair, and J. A. Dionne, Nat. Commun. 9, 1775 (2018).

[44] K. Sytwu, F. Hayee, T. C. Narayan, A. L. Koh, R. Sinclair, and J. A. Dionne, Nano Lett. 18, 5357 (2018).

[45] A. Ulvestad and A. Yau, Nat. Commun. 8, 1 (2017).

[46] B. Fultz, C. K. Witham, and T. J. Udovic, J. Alloys Compd. 335, 165 (2002).

[47] T. B. Flanagan, C. N. Park, and W. A. Oates, Prog. Solid State Chem. 23, 291 (1995). 